doi: $10.13108 / 2016-8-2-112$

\title{
RECURSION OPERATOR FOR A SYSTEM WITH NON-RATIONAL LAX REPRESENTATION
}

\author{
K. ZHELTUKHIN
}

\begin{abstract}
We consider a hydrodynamic type system, waterbag model, that admits a dispersionless Lax representation with a logarithmic Lax function. Using the Lax representation, we construct a recursion operator of the system. We note that the constructed recursion operator is not compatible with the natural Hamiltonian representation of the system.
\end{abstract}

Keywords: recursion operator, hydrodynamic type systems, non-rational Lax representation.

Mathematics Subject Classification: 17B80, 37K10, 37K30, 70H06

\section{INTRODUCTION}

In the present paper we consider the so-called waterbag model [1, [2]. This hydrodynamic type system admits a dispersionless Lax representation with a logarithmic Lax function. Such systems have important applications in the topological field theories, see [3], 4] and the references therein. For a better understanding of such systems one needs to know a biHamiltonian structure of a system and the corresponding recursion operator, see [5]-[7]. For the systems admitting dispersionless Lax representation the construction of bi-Hamiltonian structures and recursion operators is well understood in the case of a polynomial or rational Lax function [8]-[12]. The non-rational Lax functions present a much more difficult case. In the present paper we construct a recursion operator for the case of logarithmic Lax function. To our knowledge, in the literature, there are no other examples of recursion operators corresponding to a non-rational Lax function.

Let us give needed definitions. We introduce the algebra of Laurent series

$$
\mathcal{A}=\left\{\sum_{-\infty}^{\infty} u_{i} p^{i}: u_{i} \text { are smooth functions decaying fast at infinity }\right\},
$$

with the Poisson bracket given by

$$
\{f, g\}=\frac{\partial f}{\partial p} \frac{\partial g}{\partial x}-\frac{\partial f}{\partial x} \frac{\partial g}{\partial p}
$$

Taking the Lax function

$$
L=p-m \ln \left(p-c^{1}\right)+\ln \left(p-c^{2}\right)+\cdots+\ln \left(p-c^{m+1}\right)
$$

and using the Gel'fand-Dikii construction [13], we can write the hierarchy of integrable equations

$$
L_{t_{n}}=\left\{\left(L^{n}\right)_{\geqslant 0}, L\right\} \quad n=1,2, \ldots .
$$

К. ЖЕЛТУХИН, РЕКУРСИВНЫЙ ОПЕРАТОР ДЛЯ СИСТЕМ С ИРРАЦИОНАЛЬНЫМ ПРЕДСТАВЛЕНИЕМ ЛАKCA.

(c) K. Zheltukhin.

Поступила 08 декабря 2015 г. 
The second equation of the hierarchy

$$
L_{t}=\left\{\left(L^{2}\right)_{\geqslant 0}, L\right\}
$$

leads to the waterbag model

$$
c_{t}^{j}=\partial_{x}\left(\frac{\left(c^{j}\right)^{2}}{2}+m c^{1}-c^{2}-\cdots-c^{m+1}\right),
$$

where $j=1,2, \ldots,(m+1)$. As we show, the above hierarchy admits the following recursion operator

$$
\mathcal{R}=A \partial_{x}^{-1}
$$

where the matrix $A=\left(\gamma_{i j}\right)$ has the entries

$$
\begin{gathered}
\gamma_{11}=c_{x}^{1}+\sum_{j=2}^{m+1} \frac{c_{x}^{1}-c_{x}^{j}}{c^{1}-c^{j}}, \quad \gamma_{1 k}=-\frac{c_{x}^{1}-c_{x}^{k}}{c^{1}-c^{k}}, \quad \gamma_{k 1}=m \frac{c_{x}^{1}-c_{x}^{k}}{c^{1}-c^{k}}, \\
\gamma_{k k}=c_{x}^{k}-m \frac{c_{x}^{1}-c_{x}^{k}}{c^{1}-c^{k}}+\sum_{j=2, j \neq k}^{m+1} \frac{c_{x}^{k}-c_{x}^{j}}{c^{k}-c^{j}}, \quad \gamma_{k i}=-\frac{c_{x}^{k}-c_{x}^{i}}{c^{k}-c^{i}}
\end{gathered}
$$

$k \neq i$, and $k, i=2,3, \ldots, m+1$.

We observe that the above system has an obvious Hamiltonian representation with the Hamiltonian operator $\mathcal{D}=J \partial_{x}$, where $J$ is the matrix having one on the incidental diagonal and its other entries are zero.

In general, if a system has a bi-Hamiltonian representation with respect to a pair of Hamiltonian operators $\overline{\mathcal{D}}_{1}$ and $\overline{\mathcal{D}}_{2}$, one can construct a recursion operator $\overline{\mathcal{R}}=\overline{\mathcal{D}}_{2} \overline{\mathcal{D}}_{1}^{-1}$. Hence, one has $\overline{\mathcal{D}}_{2}=\overline{\mathcal{R}} \overline{\mathcal{D}}_{1}$. For systems admitting dispersionless Lax representation one can generate the whole hierarchy of Hamiltonian operators $\overline{\mathcal{D}}_{n}=\overline{\mathcal{R}}^{n} \overline{\mathcal{D}}_{1}$ [14]. It turns out that in our case, if we apply the recursion operator $\mathcal{R}$ to the Hamiltonian operator $\mathcal{D}$, the resulting operator is not Hamiltonian. Thus, the recursion operator $\mathcal{R}$ and the Hamiltonian operator $\mathcal{D}$ are not compatible. For further studies, it is an interesting open question to find a bi-Hamiltonian representation of system (1.6).

The paper is organized as follows. In Section 2 we give a construction of the recursion operator of system (1.6) for general $m$. In Section 3 we give examples of system $(1.6)$ and the corresponding recursion operator for $m=1,2,3$.

\section{Evaluation of RECURSION OPERATOR}

Let us introduce new variables

$$
c^{1}=u \quad \text { and } \quad v^{j-1}=c^{1}-c^{j}, \quad j=2,3, \ldots(m+1) .
$$

In terms of the new variables, system 1.60 becomes

$$
\begin{aligned}
& u_{t}=u u_{x}+v_{x}^{1}+\ldots v_{x}^{n} \\
& v_{t}^{1}=v^{1} u_{x}+\left(u-v^{1}\right) v_{x}^{1} \\
& \cdots \\
& v_{t}^{m}=v^{m} u_{x}+\left(u-v^{m}\right) v_{x}^{m}
\end{aligned}
$$

System (2.2) admits a Lax representation

$$
L_{t}=\left\{\left(L^{2}\right)_{\geqslant 1}, L\right\}
$$


with Lax function

$$
L=p+u+\ln \left(1+\frac{v^{1}}{p}\right)+\ln \left(1+\frac{v^{2}}{p}\right)+\cdots+\ln \left(1+\frac{v^{m}}{p}\right) .
$$

Thus, we have the whole hierarchy of the symmetries for the system 2.2 given by

$$
L_{t_{n}}=\left\{\left(L^{n}\right)_{\geqslant 1}, L\right\} \quad n=1,2, \ldots
$$

Let us construct a recursion operator for the above hierarchy of the symmetries. We construct the recursion operator by direct analysis of the Lax representation.

Let

$$
L^{n}=a_{n} p^{n}+a_{n-1} p^{n-1}+\ldots a_{1} p+a_{0}+a_{-1} p^{-1}+\ldots
$$

The next two lemmata provide some relations between coefficients of $L^{n}$ and

$$
L_{t_{n}}=u_{t_{n}}+\frac{v_{t_{n}}^{1}}{p+v^{1}}+\cdots+\frac{v_{t_{n}}^{m}}{p+v^{m}} .
$$

Lemme 2.1. For each $k=2,3 \ldots m$ and each $n=2,3, \ldots$ the identity

$$
\sum_{i=1}^{n}(-1)^{(i-1)} a_{i}\left(v^{k}\right)^{i}=\partial_{x}^{-1} v_{t_{n}}^{k}
$$

holds true.

Proof. Using (2.6) we can write the equation (2.5) as

$$
\begin{aligned}
u_{t_{n}}+\frac{v_{t_{n}}^{1}}{p+v^{1}}+\cdots+\frac{v_{t_{n}}^{m}}{p+v^{m}} & =\left(n a_{n} p^{n-1}+\cdots+2 a_{2} p+a_{1}\right)\left(u_{x}+\frac{v_{x}^{1}}{p+v^{1}}+\cdots+\frac{v_{x}^{m}}{p+v^{m}}\right) \\
& -\left(a_{n, x} p^{n}+\cdots+a_{2, x} p^{2}+a_{1, x}\right)\left(1-\frac{v^{1}}{p\left(p+v^{1}\right)}-\cdots-\frac{v^{m}}{p\left(p+v^{m}\right)}\right)
\end{aligned}
$$

Multiplying the above equation by $\left(p+v_{1}\right)\left(p+v_{2}\right) \ldots\left(p+v_{m}\right)$ and then substituting $p=-v_{k}$, we obtain

$$
v_{t_{n}}^{k}=\sum_{i=1}^{n}(-1)^{i-1} i a_{i}\left(v^{k}\right)^{i-1} v_{x}^{k}+\sum_{i=1}^{n}(-1)^{i-1} a_{i, x}\left(v^{k}\right)^{i} .
$$

That is,

$$
v_{t_{n}}^{k}=\left(\sum_{i=1}^{n}(-1)^{i-1} a_{i}\left(v^{k}\right)^{i}\right)_{x}
$$

Lemme 2.2. For each $n=2,3, \ldots$, the identity $a_{0}=\partial_{x}^{-1} u_{t_{n}}$ holds true.

Proof. Lax equation 2.5 can be written as

$$
L_{t_{n}}=-\left\{\left(L^{n}\right)_{\leqslant 0}, L\right\} \quad n=1,2, \ldots
$$

Using (2.6) and collecting coefficients of zero power of $p$ in the above equations we have $u_{t_{n}}=$ $a_{0, x}$.

The above lemmata allow us to express the coefficients of $\left(L_{>0}^{(n+1)}\right)_{p}$ and $\left(L_{>0}^{(n+1)}\right)_{x}$ in terms of coefficients of $L_{\geqslant 0}^{n}$ and $L_{t_{n}}$. 
Lemme 2.3. Let

$$
\frac{1}{n+1}\left(L_{\geqslant 1}^{(n+1)}\right)_{p}=b_{n} p^{n-1}+\cdots+b_{2} p+b_{1}
$$

Then

$$
b_{r}=a_{r-1}+\sum_{k=1}^{m} \sum_{j=0}^{r-1}\left(v^{k}\right)^{-j} a_{r-j}+\sum_{k=1}^{m}\left(v^{k}\right)^{-r} \partial_{x}^{-1} v^{k}
$$

where $r=1,2, \ldots n$.

Let

$$
\frac{1}{n+1}\left(L_{\geqslant 1}^{(n+1)}\right)_{x}=d_{n} p^{n}+\cdots+d_{2} p^{2}+d_{1} p
$$

Then

$$
d_{r}=u_{x} a_{r}+\sum_{k=1}^{m} \sum_{j=0}^{r-1}\left(v^{k}\right)^{-j-1} v_{x}^{k} a_{r-j}+\sum_{k=1}^{m}\left(v^{k}\right)^{-r-1} v_{x}^{k} \partial_{x}^{-1} v^{k},
$$

where $r=1,2, \ldots n$.

Proof. We have

$$
\frac{1}{n+1}\left(L_{\geqslant 1}^{(n+1)}\right)_{p}=\left(L_{\geqslant 0}^{(n)} L_{p}\right)_{\geqslant 0}
$$

That is

$$
\frac{1}{n+1}\left(L_{\geqslant 1}^{(n+1)}\right)_{p}=\left(\left(a_{n} p^{n}+\cdots+a_{0}\right)\left(u_{x}+\sum_{k=1}^{m} \frac{v_{x}^{k}}{p+v_{k}}\right)\right)_{\geqslant 0} .
$$

For each $k=1, \ldots m$, we expand $\frac{1}{p+v^{k}}$ as series in terms of $p^{-1}$ at $p=\infty$ and multiply with $\left(a_{n} p^{n}+\cdots+a_{0}\right)$. Collecting coefficients at $p^{k}, k=1, \ldots m$, in the above identity and using Lemma 2.1, we obtain formula (2.9). The formula (2.11) can be obtained in the same way.

Using the above lemmata, we find a recursion operator for the hierarchy 2.5 .

Lemme 2.4. The recursion operator for system (2.2) can be written as $\mathcal{R}=C \partial_{x}^{-1}$, where $C$ is an $(m+1) \times(m+1)$ matrix. It is convenient to write matrix $C$ as a sum of two matrices, $C=(A+B)$. Matrix $A=\left(\alpha_{i j}\right)$ has the entries

$$
\begin{array}{ll}
\alpha_{11}=u_{x} ; & \\
\alpha_{1(j+1)}=v_{x}^{j}\left(v^{j}\right)^{-1}, & j=1,2, \ldots, m ; \\
\alpha_{(j+1) 1}=v_{x}^{j}, & j=1,2, \ldots, m ; \\
\alpha_{(j+1)(j+1)}=\left(u_{x}-v_{x}^{j}\right), & j=1,2, \ldots, m ; \\
\alpha_{(i+1)(j+1)}=0, & i \neq j, \quad i, j=1,2, \ldots, m ;
\end{array}
$$

Matrix $B=\left(\beta_{i j}\right)$ has the entries

$$
\begin{aligned}
& \beta_{11}=0 ; \quad \beta_{1(j+1)}=0, \quad \beta_{(j+1) 1}=0, \quad j=1,2, \ldots, m ; \\
& \beta_{(j+1)(j+1)}=\sum_{k=1, k \neq j}^{m} \frac{v_{x}^{k}-v^{k}\left(v^{j}\right)_{x}\left(v^{j}\right)^{-1}}{v^{k}-v^{j}}, \quad j=1,2, \ldots, m ; \\
& \beta_{(i+1)(j+1)}=\frac{v_{x}^{i}-v^{i} v_{x}^{j}\left(v^{j}\right)^{-1}}{v^{j}-v^{i}}, \quad i \neq j, \quad i, j=1,2, \ldots, m .
\end{aligned}
$$


Proof. Using notations of Lemma 2.3 the Lax equation 2.5 can be written as

$$
\begin{aligned}
u_{t_{n+1}}+\sum_{k=1}^{m} \frac{v_{t_{n+1}}^{k}}{p+v^{k}}= & (n+1)\left(b_{n} p^{n-1}+\cdots+b_{2} p+b_{1}\right)\left(u_{x}+\sum_{k=1}^{m} \frac{v_{x}^{k}}{p+v^{k}}\right) \\
& (n+1)\left(d_{n} p^{n}+\cdots+d_{2} p^{2}+d_{1}\right)\left(1-\sum_{k=1}^{m} \frac{v^{k}}{p\left(p+v^{k}\right)}\right) .
\end{aligned}
$$

We multiply the above equation by $\left(p+v^{1}\right)\left(p+v^{2}\right) \ldots\left(p+v^{m}\right)$ and substitute the expressions for $b_{i}, d_{i}, i=1,2, \ldots n$, given in Lemma 2.3 . Equating coefficients at $p^{k}, k=1,2, \ldots m$, we obtain a system of equations linear with respect to $v_{t_{n+1}}^{k}, k=1,2, \ldots m$. Solving the system, we obtain the recursion operator given above.

Remark 2.1. Let us a define vector $V=\left(u, v^{1}, v^{2}, \ldots, v^{m}\right)$ and write system 2.2 as

$$
V_{t}=K\left(V, V_{x}\right)
$$

By straightforward calculations we check that the constructed above operator satisfies the criteria for recursion operators

$$
\mathcal{R}_{t}=\mathbb{D}_{K} \mathcal{R}-\mathcal{R} \mathbb{D}_{K}
$$

where $\mathbb{D}_{K}$ is the Fréchet derivative of $K$.

Returning back to the original variables $c^{1}, \ldots c^{m+1}$, we obtain recursion operator (1.7).

\section{EXAMPLES}

Let us consider some examples. We give examples in variables $c^{1}, c^{2}, \ldots, c^{m+1}$.

Example 3.1. Let us consider equation (1.6) with $m=1$. The equation becomes

$$
\begin{aligned}
& c_{t}^{1}=c^{1} c_{x}^{1}+c_{x}^{1}-c_{x}^{2} \\
& c_{t}^{2}=c^{2} c_{x}^{2}+c_{x}^{1}-c_{x}^{2}
\end{aligned}
$$

The above system admits the recursion operator

$$
\left(\begin{array}{lr}
c_{x}^{1}+\frac{c_{x}^{1}-c_{x}^{2}}{c^{1}-c^{2}} & -\frac{c_{x}^{1}-c_{x}^{2}}{c^{1}-c^{2}} \\
\frac{c_{x}^{1}-c_{x}^{2}}{c^{1}-c^{2}} & c_{x}^{2}-\frac{c_{x}^{1}-c_{x}^{2}}{c^{1}-c^{2}}
\end{array}\right) \partial_{x}^{-1} .
$$

Example 3.2. Let us consider equation (1.6) with $m=2$. The equation becomes

$$
\begin{aligned}
& c_{t}^{1}=c^{1} c_{x}^{1}+2 c_{x}^{1}-c_{x}^{2}-c_{x}^{3} \\
& c_{t}^{2}=c^{2} c_{x}^{2}+2 c_{x}^{1}-c_{x}^{2}-c_{x}^{3} \\
& c_{t}^{3}=c^{3} c_{x}^{3}+2 c_{x}^{1}-c_{x}^{2}-c_{x}^{3}
\end{aligned}
$$

The above system admits the recursion operator

$$
\left(\begin{array}{lll}
c_{x}^{1}+\frac{c_{x}^{1}-c_{x}^{2}}{c^{1}-c^{2}}+\frac{c_{x}^{1}-c_{x}^{3}}{c^{1}-c^{3}} & -\frac{c_{x}^{1}-c_{x}^{2}}{c^{1}-c^{2}} & -\frac{c_{x}^{1}-c_{x}^{3}}{c^{1}-c^{3}} \\
2 \frac{c_{x}^{1}-c_{x}^{2}}{c^{1}-c^{2}} & c_{x}^{2}-2 \frac{c_{x}^{1}-c_{x}^{2}}{c^{1}-c^{2}}+\frac{c_{x}^{2}-c_{x}^{3}}{c^{2}-c^{3}} & -\frac{c_{x}^{2}-c_{x}^{3}}{c^{2}-c^{3}} \\
2 \frac{c_{x}^{1}-c_{x}^{3}}{c^{1}-c^{3}} & -\frac{c_{x}^{3}-c_{x}^{2}}{c^{3}-c^{2}} & c_{x}^{3}-2 \frac{c_{x}^{1}-c_{x}^{3}}{c^{1}-c^{3}}+\frac{c_{x}^{3}-c_{x}^{2}}{c^{3}-c^{2}}
\end{array}\right) \partial_{x}^{-1} .
$$


Example 3.3. Let us consider equation (1.6) with $m=3$. The equation becomes

$$
\begin{aligned}
c_{t}^{1} & =c^{1} c_{x}^{1}+3 c_{x}^{1}-c_{x}^{2}-c_{x}^{3}-c_{x}^{4} \\
c_{t}^{2} & =c^{2} c_{x}^{2}+3 c_{x}^{1}-c_{x}^{2}-c_{x}^{3}-c_{x}^{4} \\
c_{t}^{3} & =c^{3} c_{x}^{3}+3 c_{x}^{1}-c_{x}^{2}-c_{x}^{3}-c_{x}^{4} \\
c_{t}^{4} & =c^{4} c_{x}^{4}+3 c_{x}^{1}-c_{x}^{2}-c_{x}^{3}-c_{x}^{4}
\end{aligned}
$$

The above system admits the recursion operator

$$
\begin{aligned}
& \left(\begin{array}{cccc}
c_{x}^{1} & -\frac{c_{x}^{1}-c_{x}^{2}}{c^{1}-c^{2}} & -\frac{c_{x}^{1}-c_{x}^{3}}{c^{1}-c^{3}} & -\frac{c_{x}^{1}-c_{x}^{4}}{c^{1}-c^{4}} \\
3 \frac{c_{x}^{1}-c_{x}^{2}}{c^{1}-c^{2}} & c_{x}^{2}-3 \frac{c_{x}^{1}-c_{x}^{2}}{c^{1}-c^{2}} & -\frac{c_{x}^{2}-c_{x}^{3}}{c^{2}-c^{3}} & -\frac{c_{x}^{2}-c_{x}^{4}}{c^{2}-c^{4}} \\
3 \frac{c_{x}^{1}-c_{x}^{3}}{c^{1}-c^{3}} & -\frac{c_{x}^{3}-c_{x}^{2}}{c^{3}-c^{2}} & c_{x}^{3}-3 \frac{c_{x}^{1}-c_{x}^{3}}{c^{1}-c^{3}} & -\frac{c_{x}^{3}-c_{x}^{4}}{c^{3}-c^{4}} \\
3 \frac{c_{x}^{1}-c_{x}^{4}}{c^{1}-c^{4}} & -\frac{c_{x}^{4}-c_{x}^{2}}{c^{4}-c^{2}} & -\frac{c_{x}^{4}-c_{x}^{3}}{c^{4}-c^{3}} & c_{x}^{4}-3 \frac{c_{x}^{4}-c_{x}^{1}}{c^{4}-c^{1}}
\end{array}\right) \partial_{x}^{-1} \\
& +\left(\begin{array}{cccc}
\sum_{j=2}^{4} \frac{c_{x}^{1}-c_{x}^{j}}{c^{1}-c^{j}} & 0 & 0 & 0 \\
0 & \sum_{j=2, j \neq 2}^{4} \frac{c_{x}^{2}-c_{x}^{j}}{c^{2}-c^{j}} & 0 & 0 \\
0 & 0 & \sum_{j=2, j \neq 3}^{4} \frac{c_{x}^{3}-c_{x}^{j}}{c^{3}-c^{j}} & 0 \\
0 & 0 & 0 & \sum_{j=2, j \neq 4}^{4} \frac{c_{x}^{4}-c_{x}^{j}}{c^{4}-c^{j}}
\end{array}\right) \partial_{x}^{-1}
\end{aligned}
$$

The author would like to thank Professor Maxim Pavlov for pointing out the question of constructing a recursion operator for the waterbag model and Professor Metin Gürses for fruitful discussions.

\section{REFERENCES}

1. J.H. Chang. Remarks on the waterbag model of dispersionless Toda hierarchy // J. Non. Math. Phys. 15, Suppl. 3, 112-123 (2008).

2. M.V. Pavlov. Explicit solutions of the WDVV equation determined by the 'flat' hydrodynamic reductions of the Egorov hydrodynamic chains // Preprint: nlin.SI/0606008. 2006.

3. A. Boyarskii, A. Marshakov, O. Ruchayskiy, P. Wiegmann and A. Zabrodin. Associativity equations in dispersionless integrable hierarchies // Phys. Lett. B. 515:3-4, 483-492 (2001).

4. B.A. Dubrovin. Geometry of 2D Topological Field Theories. Lecture Notes in Mathematics. 1620. Springer, Berlin (1996).

5. S.P. Tsarev. Classical differential geometry and integrability of systems of hydrodynamic type // In Applications of analytic and geometric methods to nonlinear differential equations. NATO Adv. Sci. Inst. Ser. C Math. Phys. Sci. 413, 241-249 (1993).

6. S.P. Tsarev. Integrability of equations of hydrodynamic type from the end of the 19 th to the end of the 20th century // In Integrability: the Seiberg-Witten and Whitham equations. Gordon and Breach, Amsterdam, 251-265 (2000). 
7. M.V. Pavlov. The Kupershmidt hydrodynamic chains and lattices // Int. Math. Res. Not. 2006, id 46987 (2006).

8. M. Gürses, A. Karasu and V.V. Sokolov. On construction of recursion operator from Lax representation // J. Math. Phys. 40:12, 6473-6490 (1999).

9. M. Blaszak. On the construction of recursion operator and algebra of symmetries for field and lattice systems // Rep. Math. Phys. 48:1-2, 27-38 (2001).

10. M. Gürses and K. Zheltukhin. Recursion operators of some equations of hydrodynamic type // J. Math. Phys. 42:3, 1309-1325 (2001).

11. K. Zheltukhin. Recursion operator and dispersionless rational Lax representation // Phys. Lett. A. 297:5-6, 402-407 (2002).

12. B. Szablikowski and M. Blaszak. Meromorphic Lax representations of $(1+1)$-dimensional multiHamiltonian dispersionless systems // J. Math. Phys. 47:9, 092701-092724 (2006).

13. I.M. Gel'fand, L.A. Dikii. Fractional powers of operators and Hamiltonian systems // Funkts. Anal. Pril. 10:4, 13-29 (1976). [Funct. Anal. Appl. 10:4, 259-273 (1976).]

14. L.C. Li. Classical r-matrices and compatible Poisson structures for Lax equation on Poisson algebra // Comm. Math. Phys. 203:3, 573-592 (1999).

Kostyantyn Zheltukhin

Department of Mathematics, Faculty of Science,

Middle East Technical University,

06800 Ankara, Turkey

E-mail: zheltukh@metu.edu.tr 